\title{
An Ultrasonic Line Follower Robot to Detect Obstacles and Edges for Industrial and Rescue Operations
}

\author{
Vicky Barua \\ Department of Computer Science \\ and Engineering, \\ BGC Trust University Bangladesh, \\ Chattogram, Bangladesh
}

\author{
Md. Arif Istiek Neloy \\ Department of Computer Science \\ and Engineering, \\ BGC Trust University Bangladesh, \\ Chattogram, Bangladesh
}

\author{
Md. Shafiul Islam Joy \\ Department of Electrical and \\ Electronic Engineering \\ Chittagong University of \\ Engineering \& Technology, \\ Chattogram, Bangladesh
}

\author{
Shahid Uddin Rahat \\ Department of Computer Science \\ and Engineering, \\ BGC Trust University Bangladesh, \\ Chattogram, Bangladesh
}

\author{
Nazmun Nahar \\ Department of Computer Science \\ and Engineering \\ BGC Trust University Bangladesh, \\ Chattogram, Bangladesh
}

\author{
Abhijit Pathak \\ Department of Computer Science and Engineering, \\ BGC Trust University Bangladesh, \\ Chattogram, Bangladesh
}

\section{ABSTRACT}

Line follower is a smart autonomous robot that detects or follows a visible line embedded in the ground. The trail is predetermined and can be selected with a high contrast color or with a black line visible on the trail surface. Infrared sensors are used to detect these lines. Typically speaking, the area unit of the infrared sensors is used to locate the path that the robot has to follow. The robot movement is automatic and can be used for applications of long distances. It is the fundamental line follower robot's function. The device proposed for commercial, medical, rescue and military operations are extremely useful. In particular, these past constraints are no longer necessary with recent technological advances in computing. The production of tracking systems can now be made more capable of reliably estimating the target location behind the obstacle. The benefit of these technologies consists in the possibility of using an ultrasonic method for measurement without direct contact with a target. Different models and systems for indoor and outdoor object detection have been described in the literature. Using optical, heat base, infrared and ultrasonic approaches, object localization techniques were introduced. Indoor positioning systems monitor and locate objects and enclose environments inside buildings. Wireless methods, optical tracking, and ultrasonic techniques are used for object position detection systems. The goal of this study is to develop a monitoring system that follows certain paths and can detect objects and edges using ultrasonic frequencies. If some object is put, a regular line follower will try to move and smash the obstacle. This prototype of line follower robot tries to push the limit little to overcome this issue. It has been built in a way that any obstacle in front of it can be identified. It will stop and will not pass until the barrier remains. Also, it is able to identify every front edge and comply similarly. In industries, such as material handling, this type of robot performs many tasks.
\end{abstract}

These robots are also used as machine-controlled carrier instruments in old conveyor belts switching industries.

\section{General Terms}

Robotics, Internet of Things (IoT), Programming

\section{Keywords}

Line Follower; Edge Detection; Obstacle Detection; Ultrasonic Sonar Sensor; IR Sensor; Autonomous Robot.

\section{INTRODUCTION}

Robotics is a development segment dedicated to the design, building, service, production, and deployment of robots. Robotics is concerned with the electronics, engineering, mechanics, and software sciences. In robotics, avoidance of obstacles is the task of achieving some goal of the control subject to constraints on the location of non- intersection or non-collision. Obstacle avoidance is one of the key research areas and is also a foundation for a construction robot's successful results. Because of its high efficiency and reliability Robotics is now commonly used in many industries. All mobile robots are designed to avoid obstacles. The architecture of a stand-alone robot needs several sensors and actuators to be configured according to their tasks. Every autonomous robot must identified obstacles primarily. The main reason why this system is used for transporting goods is that it fits and forgets that the machine's operation is fully automatic after the device is set down on the specified path. The movement of a robot to sense obstacles and edges is entirely autonomous. Because of these factors, the process of following the line has many useful applications. A standard line following mechanism is built using low-cost equipment to locate obstacles and edge. The device will use a low-cost tracking mechanism. It increases the practical nature of the line tracking device as obstacles in any working environment 
are natural and if the line tracker cannot meet any obstacle on its way it crashes and can be seriously harmed. The robot receives data from its environment via robot-based sensors. For avoiding obstacles, various types of sensors can be used. The avoidance methods depending on the use of the sensor are distinct. Some robots accomplish the detection with a single sensing device. However, several other robots use several sensors. The edge detection module is constructed using the IR module for line detection. The modules are attached to the BOT to sense the edge at an early stage and function correctly. Because of the rotation radius of the wheels, the distance between sensors is also greater than the width of the BOT. The IR module gives the controller a high pulse when the surface is detected and when the edge is detected the IR module does not reflect the light so that the controller has a low pulse. The widely used sensors for preventing obstacles include bump sensors, infrared sensors, ultrasound sensors, laser distance detectors, web camera cameras with CCD couplers, etc. These include an ultrasonic sensor which is best suited to avoid the robot, due to its low cost and range. This smart device can also be used for health care monitoring in hospitals, which reduces the human effort of patient evaluation and medical supplies and items. The workers are not used to move goods from one location to an alternative but to use this strong and knowledgeable line follower method to perform alternative tasks.

\section{LITERATURE REVIEW}

This Line Following Robot, which is a part of AGV (Automated Guided Vehicle), an autonomous robot in a simple term, can detect black or white lines to follow them. Students have been developing this kind of robots for a while. But the basic concept has always been the same [1]. And line follower robot competition held commonly to encourage students more about it [5]. These robots determine the black or white line using Infrared rays. And makes its path following that line. As the robot works fully autonomously engineers have tried to bump up its abilities to make it more reliable for practical usage in various sectors. Making it capable of avoiding obstacles [2].

This Line Following Robot, which is a part of AGV (Automated Guided Vehicle), the autonomous robot in a simple term, can detect black or white lines to follow them. But research has been done to make them capable of following any color [3]. Light sensors based on LED or LDR and RFID based verification systems have been used to upgrade the line follower robot to that level.

How a Line Following robot runs is based on the sensor value it gets from the sensors. The correct sensor value leads to the correct result. That's why it's important to have an accurate idea of those sensor data and so on. That is what the researchers have done. They not only built their line follower robot prototype but also went through those data. Based on which the robot takes its decisions [4].

Path planning is the most crucial part of the line follower robot. As it is autonomous, it has to make the decision based on the path. Most robots can follow a straight or round-shaped path. However, vital part is to let it take its decision when there is more than one path to go and they are of different shapes obviously [6]. A practical study has been done to implement this in the prototype model.

Speaking of solving practical life problems using line follower robots, in our residences video surveillance cameras are so common. One drawback of them is it is stuck in one place. The authors of this paper propose that these surveillance cameras can be used with line follower robots [7]. In this way, it will have a bigger coverage.

The best concept we want for a line follower robot is, it should be able to travel through the most critical path in the least possible time. Such as we can mention T shape path, complicated loops, and critical angles and so on. These things lead a line follower robot to be perfect. But we want to use the minimum sensors possible to keep the price low. Researchers have gone through all these to make the perfect Line Following Robot [8].

\section{MATERIALS AND METHODS}

\subsection{Prototype Design}

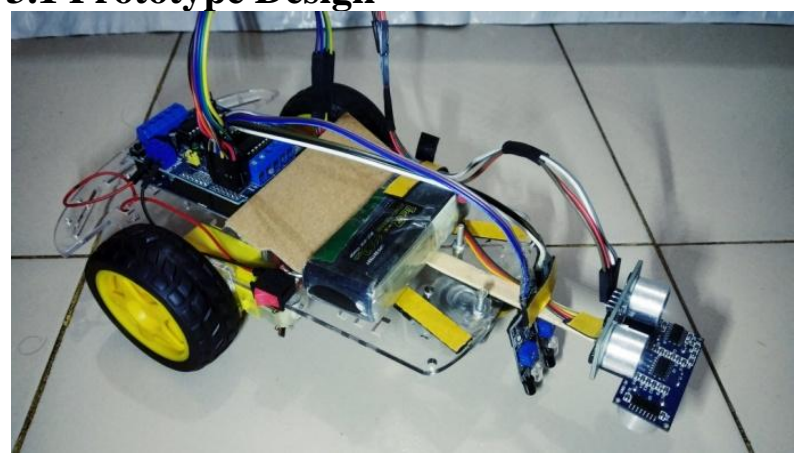

Figure.1. Snapshot of the Prototype

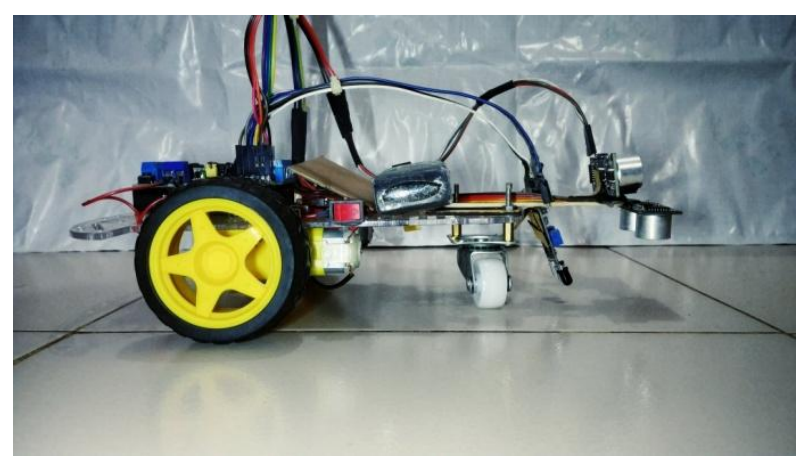

Figure.2. Snapshot of the Prototype

The hardware needed to finish this project is as follows:

- $\quad$ Arduino Uno R3

- Motor Shield L293D

- 2x DC Motors

- 2x Ultrasonic Sonar Sensor HC-SR04

- 2xIR Sensor SEN-00112

- $\quad$ 11.1V 3S 1200mAh Li-Po Battery

Here the L293D motor shield was used to detect line also prevent edge and setback path. Here in this line follower robot two IR sensors modules have been used, left sensor and right sensor. They have been used to detect the black line. And it uses two ultrasonic sensors. One of the ultrasonic sensors is facing in front to detect the obstacle and another one is facing down to detect edges on the surface. When both left and right sensor senses black then the robot moves forward (Figure. 2). As long as it is getting a measurable black line it is moving forward. If the ultrasonic sensor detects the obstacle placed on the black line, then the robot stops. (Figure. 3). It will keep standing there as long as the obstacle is present. If another ultrasonic sensor detects the edge, then the robot stops as well 
(Figure. 4)

\subsection{Line Following Principle}

Here in this line follower robot, we are using two IR sensor modules, left sensor and right sensor and two ultrasonic sensors. One of the ultrasonic sensors is facing in front to detect the obstacle and another one is facing down to detect edges on the surface. When both left and right sensor senses black then the robot moves forward (Figure. 3). As long as it is getting a measurable black line it is moving forward. If the ultrasonic sensor detects the obstacle placed on the black line, then the robot stops. (Figure. 4). It will keep standing there as long as the obstacle is present. If another ultrasonic sensor detects the edge, then the robot stops as well (Figure. 5).

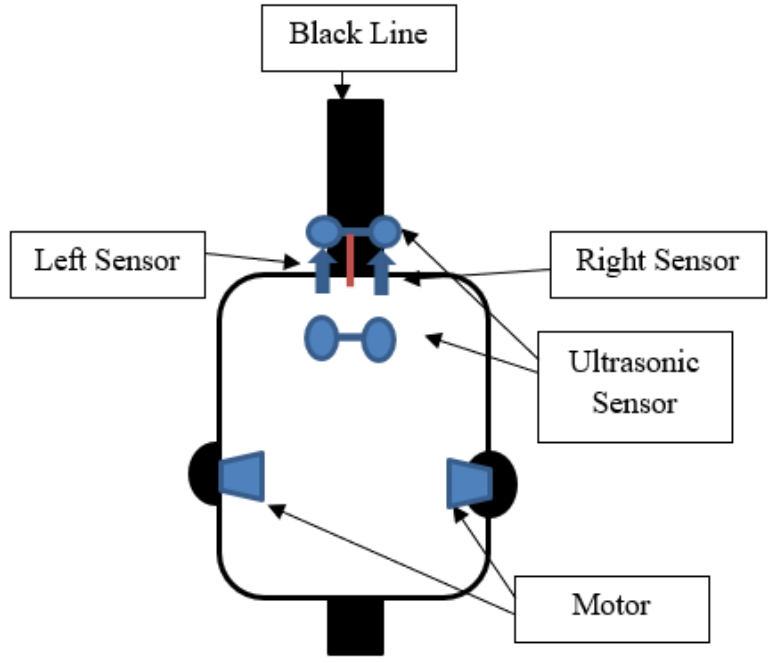

Figure.3. Moves Forward

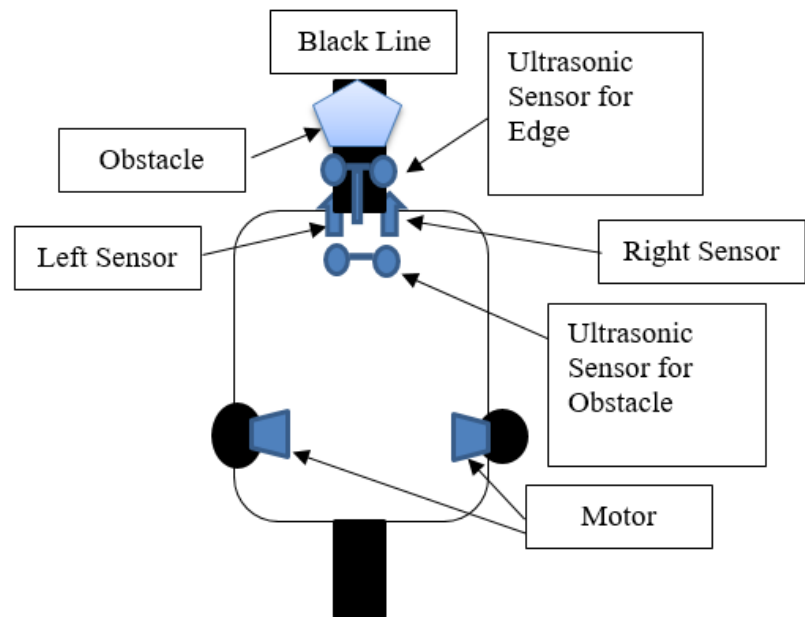

Figure.4. Motor Stops for Obstacle

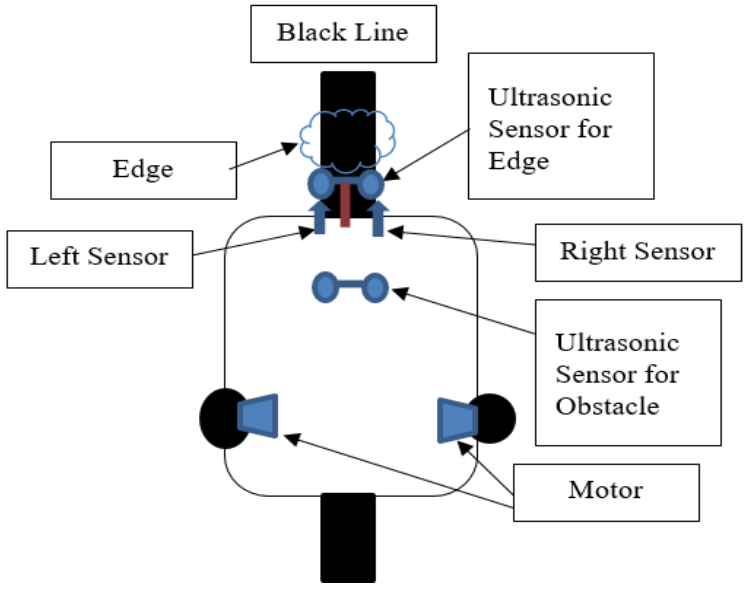

Figure.5. Motor Stops for Edge

\subsection{Paths of Line Follower}

Robot line follower leads the track traced on the ground. The line is mostly black on a white surface. If there's no barrier or edge on its path, the robot keeps moving forward. If the robot has any obstacle or edge on the way, it will stop. Using programming code the movement of the robot can easily be modified for different paths. The robot will follow $45^{\circ}$ and in a bad angle, loop or bad curves. If it sees a crossing of a black line it stops. 


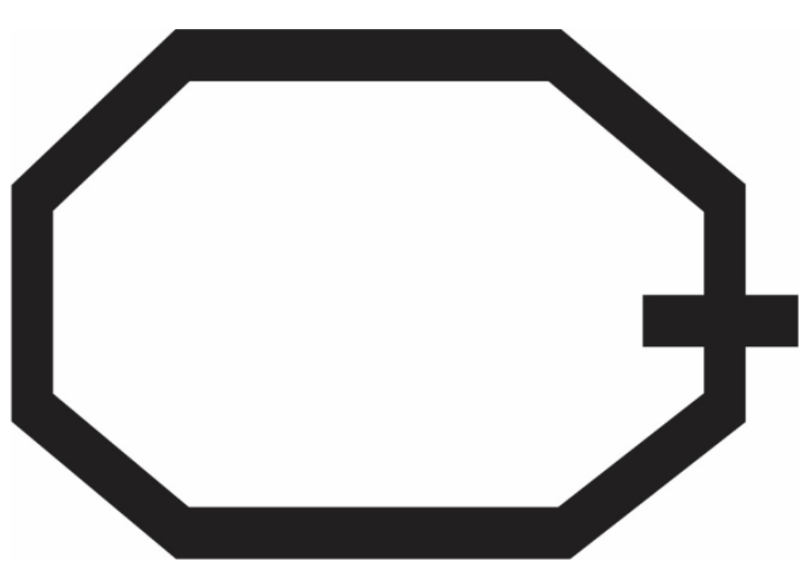

Figure.6. Polygon Shape Path

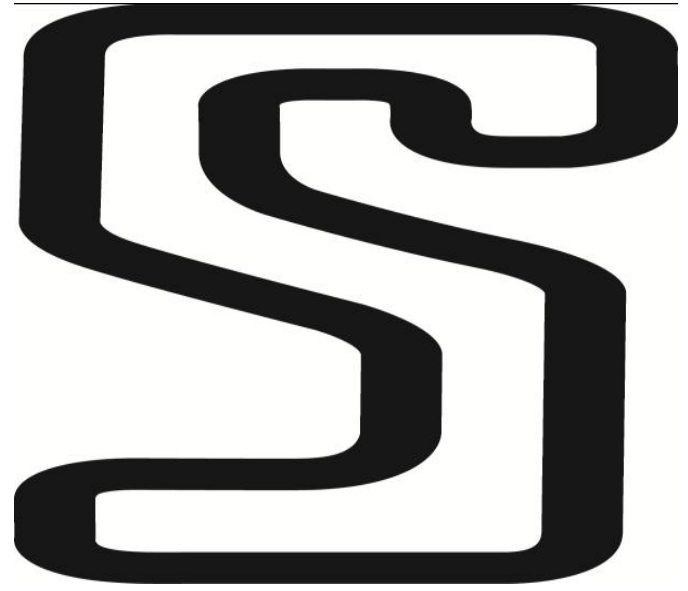

Figure.7. Hybrid Path

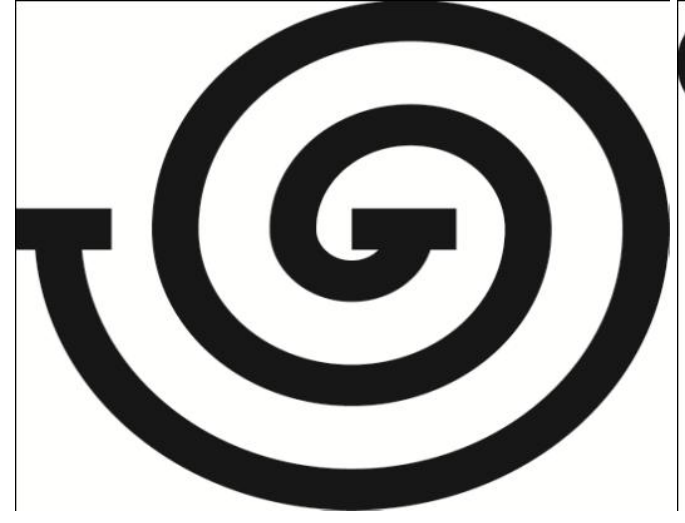

Figure.8. Spiral Path

\subsection{Block Diagram}

Here the block diagram of the system is shown in Figure- 10 . It shows that the Arduino Uno R3 sends a signal to the L293D Motor Shield. It tries to use the Motor Shield L293D with full of its potential. All kinds of processing takes place in the Arduino Uno R3 microcontroller. Two Dc Motors are

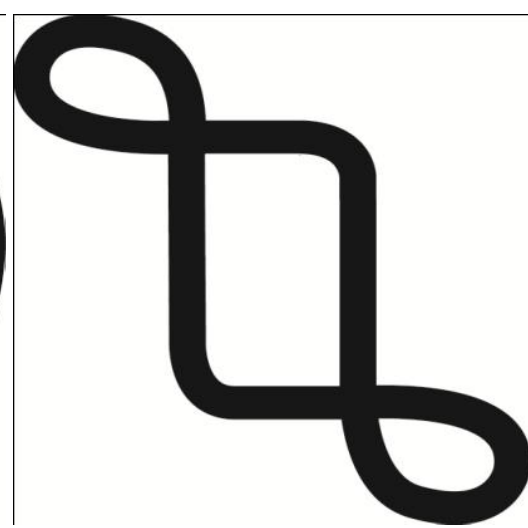

Figure.9. Loop Path

connected to the motor shield, which is the main purpose of using it. Two IR sensors and two Ultrasonic Sensors are connected to the motor shield as well. They send signals through the motor shield to the Arduino. The power source for the whole system, 11v Lithium Polymer battery is connected to the motor shield.

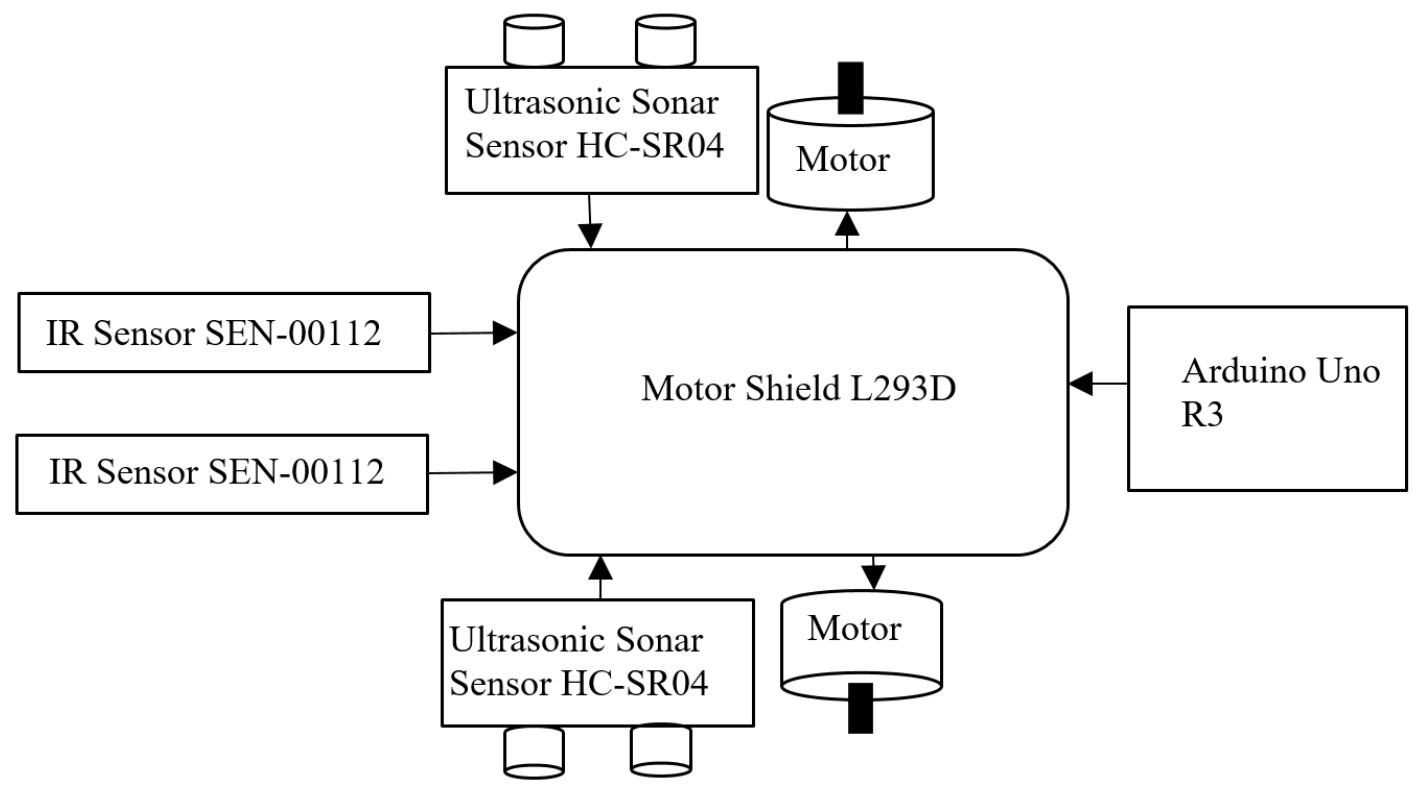

Figure.10. Block Diagram of the System 


\subsection{System Architecture}

Here the L293D Motor Shield has been used to detect line as well as also to prevent edge and setback path. It's a kind of interface between Arduino and dc motors. It is capable of handling 4 Dc Motors at a time. But for this robot, only two of these motors have been used. And this L293D Motor Shield can also power 2 servo motors at a time. But for this robot, it doesn't need any of these. The motor 1 connected with M1 slot and motor 2 connected with the M2 slot of the motor shield. Then the trig pin of first Ultrasonic Sensor is connected to $\mathrm{A} 0$ and echo pin is connected to $\mathrm{A} 1$, for second Ultrasonic Sensor trig pin is connected to A2 and echo pin is connected to A4. All ground and VCC pins from both ultrasonic sensors are attached to the ground and $5 \mathrm{~V}$ pin of the motor shield respectively. Then OUT pin of two IR sensors is connect to A3 and A5 respectively. The 11V Lithium Polymer battery has been used to power the entire unit, is attached to the external power supply terminal of the motor shield. The motor shield, however, requires external power supply, and no additional power is required for the Arduino itself.

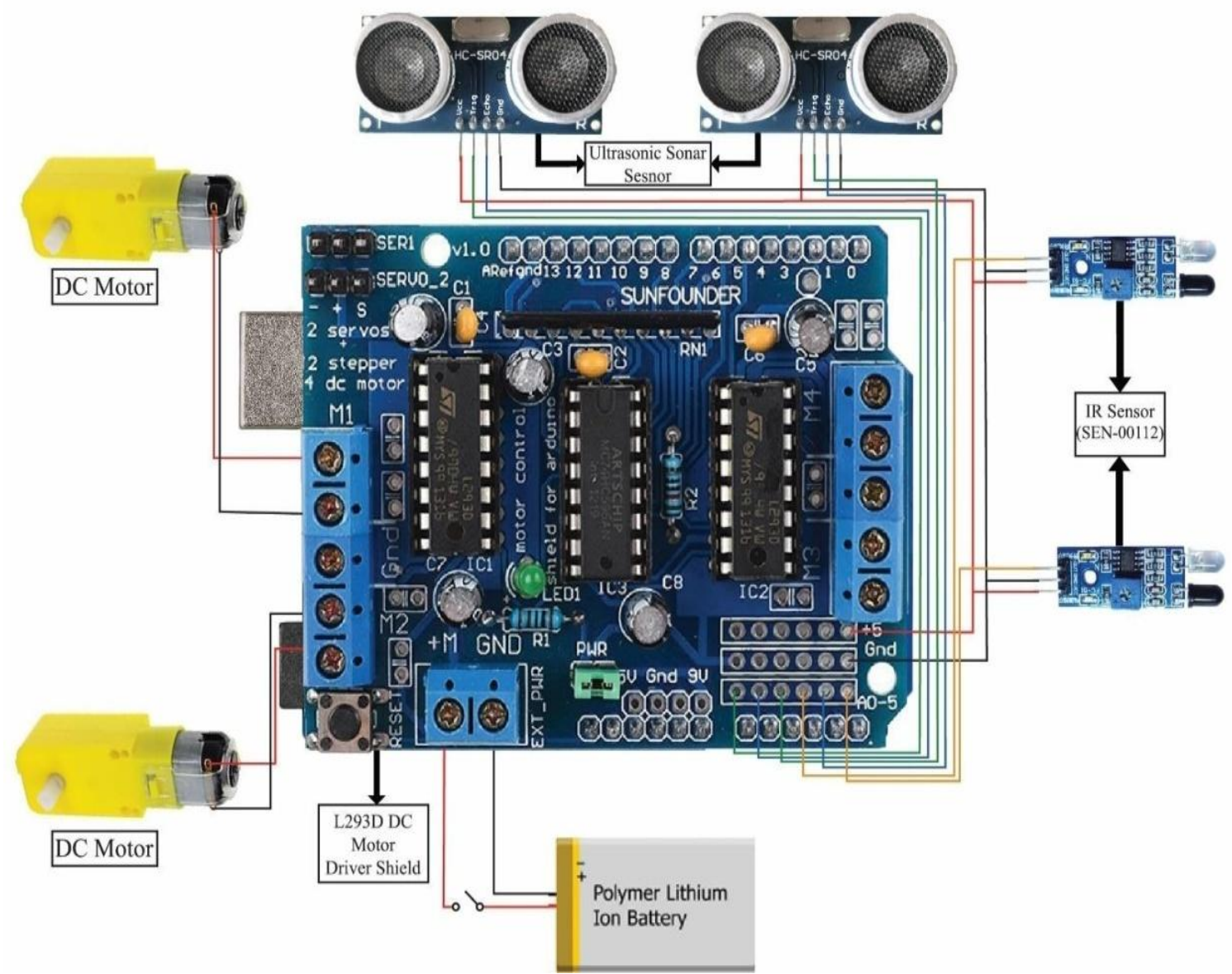

Figure.11. Architecture of the System

\subsection{Algorithm}

Step 1: Start

Step 2: Initialization

Step 3: Is there any black line?

a) If yes, go to step 4

b) If no, go to step 7 Step 4: Is there any obstacle or edge?

a) If yes, go to step 7

b) If no, go to step 5

Step 5: Move forward following the black line

Step 6: Go to step 3

Step 7: Stop

\subsection{System Flowchart}

In this flow chart, it shows the decision making process of the robot. First of all, the robot will not start the movement after turning on the power. The first thing it will do is to search for a black line. If the robot finds the black line, then it will move forward. When it's way to moving forward by following the black line it will search for obstacle and edge in its path in every moment. It will smoothly move forward whenever the black line is available and if there is no obstacle or edge. If the black line is not available, it will stop. When black line is available but the robot faces any obstacle or edge, then it will immediately stop. If the black line is available and the obstacle is removed with no edge, then the robot will continue to move forward again. Finally, if the power is down, then the robot will completely stop. 


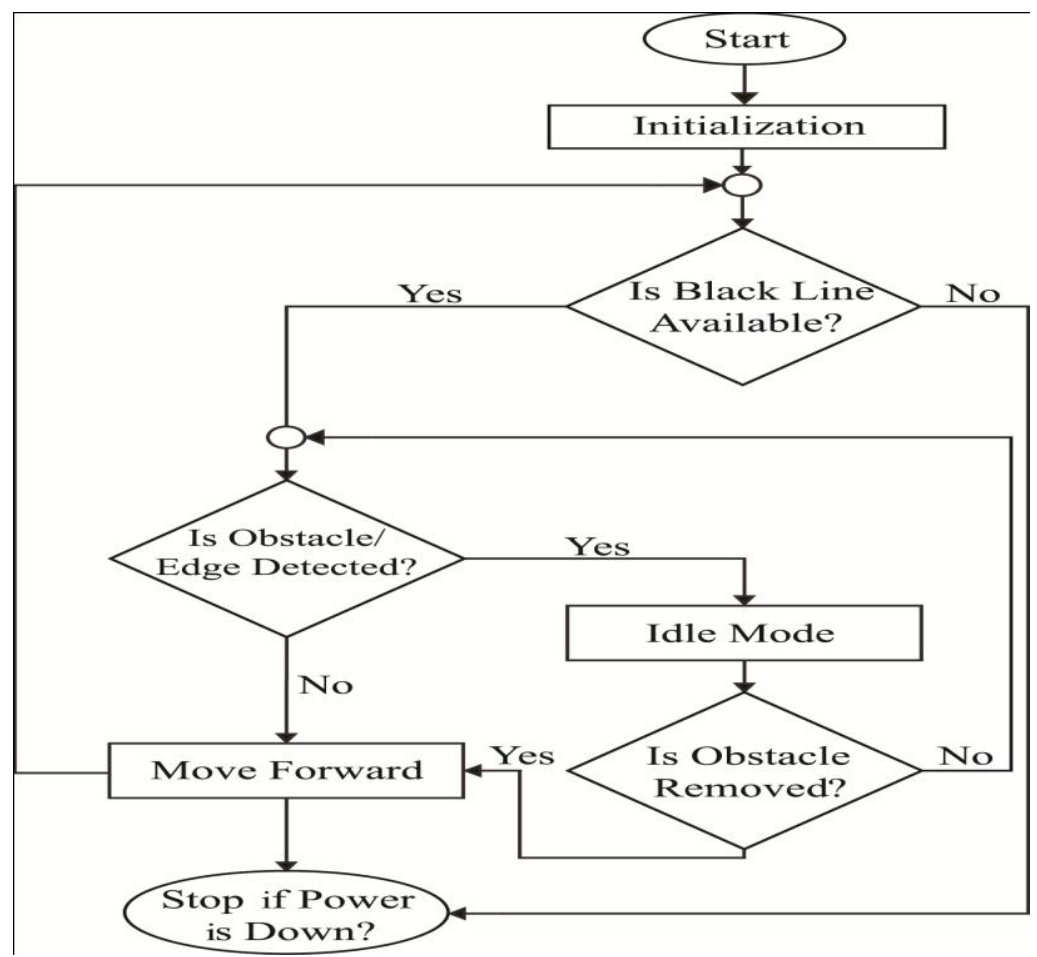

Figure.12. Flowchart of the System

\section{DISCUSSION}

\subsection{Applications}

- In restaurants or hotels, the line follower robot can be used to serve foods to the customers' table. So, the waiters don't have to carry the food anymore. By simply inputting the table number in the robot and it will carry it to the customer following the path.

- In terms of infected patients, specifically, virus-infected patients, it is always risky to reach them as a firstperson. But it is also important to offer them their medicinal products on time at the same time. A line follower robot can come handy in these kinds of situations. As it can treat certain patients without the supervision or control of any human being. It can easily follow the lines through the cabins and reach medicines to the infected patients to their cabin and return safely.

- This line follower robot can be used in industries or factories to automate the transport of parcels or goods from one place to another, using the crane system.

- This line follower robot can be used to pick up material from the mine. It is the position in which the edge mechanism of the robot sets in. The mine is full of impediments and holes. In this case the robot will use the tool for the detection of obstacles and edges to pick up materials from the mine quickly.

\subsection{Limitations}

The applications of the line follower are limited since they can't be handled. The only way to keep the followers of the line in charge is to change the course. This robot can't take any turn that is 90 degrees or smaller than that. The module that we used the SEN-00112 IR sensor is not capable of working in more lighting conditions. Besides these drawbacks, smart and intelligent line follower with edge and obstacle robots with a predefined path can be used for long- distance applications.

\subsection{Future Works}

This work is all about Line Follower and Obstacle and Edge Detection Robot using Arduino which will follow a specific line or route and avoid the obstacles and edge it encounters. By attaching the Bluetooth module and a camera, this project can be expanded in the future, so that the user can see the obstacle identified on his screen by sitting at just one spot. Line follow and obstacles with the robot industrial manufacturing processes for edge detection and medical emergency supply in Bangladesh will play a vital role in the industry. This line following and obstacle and edge detection robot can be used as carrying the load to transport the goods smoothly and without damage from one position to another. When some kind of mishandling of products happens then that device will interrupt its routine operation and call the device administrator to check the issue that occurred to fix. A Wi-Fi system, GPS device, and Camera could be used for this purpose to track the production process and the supply chain in real-time. Every industry or medical authority's practical job can be more effective for supply chain management, so that Bangladesh's manufacturing sectors take place in foreign markets.

\section{CONCLUSION}

Robotics play a significant part in the global economy and everyday life. Another challenge of robotics research is to be successful and to develop patents according to the complexity of their applications for global industries. The market for robotics technology is rising in a wide variety of applications and human activities, particularly for the manufacturing, medical, utility, defense and consumer industries. This line follower and obstacle with edge detecting robot is the prototype of robots for industrial use. This smart and intelligent robot has more benefits because it doesn't consume much power. Our project aims at creating an autonomous robot that intelligently senses the obstacle and the edge in its path and navigates according to the behavior that we have set 
for it. So, what this system provides is an alternative to the existing system by replacing skilled labor with robotic machinery, which in turn can handle more patients in less time with better accuracy and a lower cost. Performance can be improved by using good materials and great sensing power also improves motor movement. The setup cost of the line follower robot depends primarily on the costly machinery, property, and building and staff around the clock to maintain and use the machinery. In Bangladesh with a humongous population and limited capital.

\section{REFERENCES}

[1] Cao Quoc Huy, "Line Follower Robot", University UPG din Ploiesti.

[2] Chowdhurya, Nakib Hayat, Deloara Khushib, and Md Mamunur Rashidc. "Algorithm for line follower robots to follow critical paths with minimum number of sensors." International Journal of Computer 24, no. 1 (2017): 1322.

[3] F. Kaiser, S. Islam, W. Imran, K. H. Khan and K. M. A. Islam, "Line follower robot: Fabrication and accuracy measurement by data acquisition," 2014 International Conference on Electrical Engineering and Information \& Communication Technology, Dhaka, 2014, pp. 1-6.

[4] Gao Y., Fu G., Nieto A. A comparative study of gas explosion occurrences and causes in China and the United States. Int. J. Min. Reclam. Environ. 2015; 30:269-278. doi: 10.1080/17480930.2015.1043770.

[5] K. M. Hasan, A. Al-Nahid, K. J. Reza, S. Khatun and M. R. Basar, "Sensor based autonomous color line follower robot with obstacle avoidance," 2013 IEEE Business Engineering and Industrial Applications Colloquium (BEIAC), Langkawi, 2013, pp. 598-603.

[6] Kahe, "AVR Microcontroller", Nas Publication, 2007.

[7] Kasprzyczak L., Trenczek S., Cader M. Robot for monitoring hazardous environments as a mechatronic product. J. Autom. Mob. Robot. Intell. Syst. 2012; 6:5764.

[8] M. B. Nugraha, P. R. Ardianto and D. Darlis, "Design and implementation of RFID line-follower robot system with color detection capability using fuzzy logic," 2015 International Conference on Control, Electronics, Renewable Energy and Communications (ICCEREC), Bandung, 2015, pp. 75-78.
[9] M. Mashaghi, "Robotic Guide", Kanone Oloum Publication, 2008.

[10] M. Pakdaman and M. M. Sanaatiyan, "Design and Implementation of Line Follower Robot," 2009 Second International Conference on Computer and Electrical Engineering, Dubai, 2009, pp. 585-590.

[11] M. Pakdaman, M. M. Sanaatiyan and M. R. Ghahroudi, "A line follower robot from design to implementation: Technical issues and problems," 2010 The 2nd International Conference on Computer and Automation Engineering (ICCAE), Singapore, 2010, pp. 5-9.

[12] M.Zafri Baharuddin, "Analyst of Line Sensor Configuration for Advanced Line Follower Robot", University Tenaga Nasional.

[13] Murphy R., Kravitz J., Stover S., Shoureshi R. Mobile robots in mine rescue and recovery. IEEE Robot. Autom. Mag. 2009; 16:91-103. doi 10.1109/MRA.2009.932521.

[14] P. Heyrati, A. Aghagani, "Science of Robot Disgn and Build Robot", Azarakhsh Publication, 2008.

[15] Reddy A.H., Kalyan B., Murthy C.S. Mine Rescue Robot System-A Review. Procedia Earth Planet. Sci. 2015; 11:457-462. doi: 10.1016/j.proeps.2015.06.045.

[16] Robotic Competition Rolls site: http://www.tjrc.ir/roles.asp in date 2009/05/15.

[17] Saad, Wira Hidayat Mohd, Saiful Anuar Abd Karim, Norliana Azhar, Zahariah Manap, Yew Yuan Soon, and Masrullizam Mat Ibrahim. "Line Follower Mobile Robot for Surveillance Camera Monitoring System." Journal of Telecommunication, Electronic and Computer Engineering (JTEC) 10, no. 2-7 (2018): 1-5.

[18] Sure, Ramshetty K., and Savita Patil. "Android Based Autonomous Coloured Line Follower Robot." International Journel of Research and Technology 3 (2014).

[19] Thrun S., Hahnel D., Ferguson D., Montemerlo M., Triebel R., Burgard W., Baker C., Omohundro Z., Thayer S., Whittaker W. A System for Volumetric Robotic Mapping of Abandoned Mines; Proceedings of the 2003 IEEE international Conference on Robotics and Automation; Taipei, Taiwan. 14-19 September 2003; pp. $4270-4275$ 